\title{
Towards Norm Realization in Institutions Mediating Human-Robot Societies
}

\author{
Alicja Wasik, Stevan Tomic, Alessandro Saffiotti, Federico Pecora, Alcherio Martinoli and Pedro U. Lima
}

\begin{abstract}
Social norms are the understandings that govern the behavior of members of a society. As such, they regulate communication, cooperation and other social interactions. Robots capable of reasoning about social norms are more likely to be recognized as an extension of our human society. However, norms stated in a form of the human language are inherently vague and abstract. This allows for applying norms in a variety of situations, but if the robots are to adhere to social norms, they must be capable of translating abstract norms to the robotic language. In this paper we use a notion of institution to realize social norms in real robotic systems. We illustrate our approach in a case study, where we translate abstract norms into concrete constraints on cooperative behaviors of humans and robots. We investigate the feasibility of our approach and quantitatively evaluate the performance of our framework in 30 real experiments with user-based evaluation with 40 participants.
\end{abstract}

\section{INTRODUCTION}

Social norms are a fundamental part of human society. Norms create prescriptions for social behaviors, facilitate decision making in unknown situations and regulate how humans communicate, act and cooperate during social engagements. For the robots to become integrated into our society, they will need to be aware of the social norms and able to act according to the expectations that the social norms create. Better understanding of the norms can lead to a higher acceptance of robots in our everyday lives [1].

Norms of human societies are formulated in human language and as such, they share problems of lack of clarity [2]. Even for humans, interpreting rules is more challenging than writing them down. For robots that operate on robotunderstandable commands, interpretation of norms defined in human language is close to impossible. The problem of norm concretization has been identified in fields ranging from Economics [2], which focuses on the classification of norms rather than their concretization, to Computer Science [3], predominantly within the field of Multi-Agent

Supported by ISR/LARSyS Strategic Funds from FCT project FCT[UID/EEA/5009/2013] and FCT/11145/12/12/2014/S and the European Commission Horizon2020 Research and Innovation Programme under grant agreement No. 737858 (CARESSES).

Pedro Lima is with the Institute for Systems and Robotics, Instituto Superior Técnico, Universidade de Lisboa, Portugal; paleisr.ist.utl.pt

Alicja Wasik and Alcherio Martinoli are with the Distributed Intelligent Systems and Algorithms Laboratory, School of Architecture, Civil and Environmental Engineering, École Polytechnique Fédérale de Lausanne, Switzerland; alicja.wasikeepfl.ch and alcherio.martinolidepfl.ch

Stevan Tomic, Alessandro Saffiotti, and Federico Pecora are with Centre for Applied Autonomous Sensor Systems, Örebro University; tomic01@gmail.com, asaffiodaass.oru.se and federico.pecoradoru.se
Systems (MAS). Formalization of norms in MAS usually takes some form of deontic logic, but it is hard to directly connect this kind of norms with the practice as norms are formulated in a very abstract way [3], where there is no precise connection between the abstract normative statements and any computational model. As pointed out in [4], clear operational semantics is required in order to achieve computational frameworks. Existing frameworks, including ISLANDER [5], MOISE [6] and OPERA [7] are disconnected from physical execution and cannot easily be applied to robots.

Conversely to the field of MAS, some works in the field of robotics put social norms into practice [8], but these are often domain specific and do not have abstract, reusable models. Current research in robotics largely treats norms as prescriptions for behavior designers on how to select or modify robot behaviors. Norms are used for designing what the robots should do [9] [10], but not for how to do it. To address the question of how to shape robot behaviors according to social norms, the majority of social robotics research refers to the models of Proxemics [11] and Social Forces [12], based on which norms are implicitly used for social path planning [13][14], human guidance [15] and behavior selection [16]. Lists of descriptive norms written in a natural language are employed in [17], [18] and [19], but the translations of norms are method-specific, and target primarily single-robot navigation methods but put little emphasis on robot-robot or robot-human cooperation. Collective norms for cooperative behaviors have been proposed in [20] but with no systematic formalization. In summary, the current research largely treats norms as prescriptions for behavior designers on how to select or modify the robot behaviors, and the robots have no choice but to follow those prescriptions. Although the main purpose of norms is to guide social interaction, no systematic framework exists to this day that could introduce the normative aspect to multi-human, multi-robot behaviors.

In our human societies, norms are strongly inherent parts of institutions, which additionally to the traditional view of formal organizations, include abstract concepts such as family, language or education. By enforcing norms that influence human behavior [21][22], institutions are devices for reducing uncertainty, simplifying decision making and promoting cooperation [2]. In the emergent field of Institutional Robotics (IR) [23], institutions are introduced as coordination devices in multi-robot systems to facilitate the integration of robots in human societies. IR has been exploited in the context of purely robotic systems [24] and for coordinating human-robot formations [20][25], but only in simulation. 
With our formal framework based on the notion of institutions, in [25][26] we proposed a clear separation of norms from concrete physical systems, thus allowing to specify norms that are abstracted from the systems they regulate. Social norms and the context in which they operate are encapsulated in the notion of institution, which is reusable across situations. In this work, with norm realization, we provide the means to interpret vague norms in terms of robotunderstandable language, readily implementable onto concrete restrictions of robot behaviors. We apply our formalism to cooperative behaviors to show how abstract social norms become implementable in real physical systems and how the same social norms make allowances for establishing mixed human-robot formations in real environments with human participants. To the best of our knowledge, this is the first study where multiple humans and multiple robots engage in coordinated navigational behaviors. Our case study has been introduced in [25] in simulation, while in this work we validate our methods in experiments with human participants.

The rest of this paper is organized as follows. Section II outlines our formal framework for institutions. Section III shows how, using our framework, we can realize abstract norms in real physical systems. Sections IV and V describe a case study that illustrates this process and present experiments with user studies. Section VI concludes.

\section{GROUNDED InSTITUTIONS}

We start by summarizing the main concepts of our formal framework for grounded reasoning with institutions [26].

\section{A. Institutions}

Our institutions encapsulate a collection of norms together with the roles, actions and artifacts that they refer to. We define the following sets: Roles, Acts and Arts. A role in Roles is a function assigned to an agent, such as to be a Leader of a group. Acts are actions that have to be taken when playing a role, for example, the Leader might have to Guide its team. Actions are carried out with artifacts in Arts, that might have a physical or virtual realization. One of the artifacts of the Guide action of the Leader is the Destination to which the team is guided.

Norms, similarly as in human institutions, are inherently vague statements [3], general enough to be interpreted concretely in diverse situations. Norms take the form of a human readable sentence with a specific syntax that includes a natural separation between subject, verb and object. In terms of the institutional elements, roles are taken by the subject, actions are the verbs and the artifacts are the objects. Then it is natural to define the norms as predications over statements, where a statement is a relation between a subject (role), a predicate (action) and an object (artifact):

Definition 1: A norm is a statement where a qualifier forms a relation between Roles, Acts and Arts.

For example, a norm can be a statement "Leader should Guide the team towards the Destination when all the members of the team are following". Our definition of norm is similar to [2], where the qualifiers comprehend the deontic expressions, and roles, actions and artifacts are equivalent to attributes, aims, and conditions, respectively.
Institutions put all the above elements together:

Definition 2: An institution is a tuple

$$
\mathcal{I}=\langle\text { Arts, Roles, Acts, Norms }\rangle \text {. }
$$

\section{B. Domains}

An institution is an abstraction, which can be instantiated in concrete systems that are physically different but are described by the same structure. The systems are given semantics understandable by all the agents that operate within them, humans and robots. We call such a concrete system a domain.

Definition 3: A domain is a tuple $\mathcal{D}=\langle A, O, B, F, R\rangle$ :

- $A$ is a set of agents,

- $O$ is a set of physical or virtual objects,

- $B$ is a set of behaviors,

- $F \subseteq A \times B \times(O \cup A)$ is a set of affordances,

- $R$ is a finite set of state variables.

The set $A$ can include humans and robots. $B$ is the collection of all behaviors that these humans or robots can perform. $O$ are physical or virtual objects in the domain recognized by all agents. We refer to the subset of the virtual objects as knowledge $K \in O$. Knowledge gives the agents the means to comply with the norms. For example, any agent moving in human-populated environments should know about the maximal allowable speed, the value of which is further enforced by the norms. The affordance relation $F$ indicates which agents can execute which behaviors and on which objects. The state variables $R$ define properties pertaining to the objects in the domain. They may indicate the position of an agent or an object, the activation of a behavior, etc.

Definition 4: Given a domain $\mathcal{D}=\langle A, O, B, F, R\rangle$, the state space of $\mathcal{D}$ is $\mathcal{S}=\prod_{\rho \in R} \operatorname{vals}(\rho)$, where vals $(\rho)$ are the possible values of state variable $\rho \in R$. We call any element $s \in \mathcal{S}$ a state. The world state at time $t$ is $\mathcal{W}_{t}$.

Furthermore, conditions $C$ are the results of evaluating Boolean functions $f: R \rightarrow\{$ True, False $\}$ over state variables $R$. For example, if a state variable agent. type = robot, then the condition can_sleep(agent. type) is False.

\section{Grounding}

Grounding provides the key to reuse the same abstract institution to describe or regulate different systems.

Definition 5: Given an institution $\mathcal{I}$ and a domain $\mathcal{D}$, a grounding of $\mathcal{I}$ into $\mathcal{D}$ is a tuple $\mathcal{G}=\left\langle\mathcal{G}_{A}, \mathcal{G}_{B}, \mathcal{G}_{O}\right\rangle$, where:

- $\mathcal{G}_{A} \subseteq$ Roles $\times A$ is a role grounding,

- $\mathcal{G}_{B} \subseteq$ Acts $\times B$ is an action grounding,

- $\mathcal{G}_{O} \subseteq$ Arts $\times O$ is an artifact grounding.

Grounding plays an important role in our framework, by establishing the relation between an abstract institution and a specific domain. It relates roles to agents, generic actions to behaviors of agents, and institution artifacts to physical or virtual objects. While the grounding of roles, actions and physical objects allows for using institution in a concrete system, grounding of artifacts to virtual objects provides a simple, but powerful way to customize agent behaviors. For example, an artifact representing a PersonalSpace (i.e. Proxemics) of agent Wally can be grounded to ellipsoidal area with dimension that reflects Wally's expected preferences.

Next, abstract norms are realized to act on concrete behaviors of concrete robots in a real physical system using norm realization. 

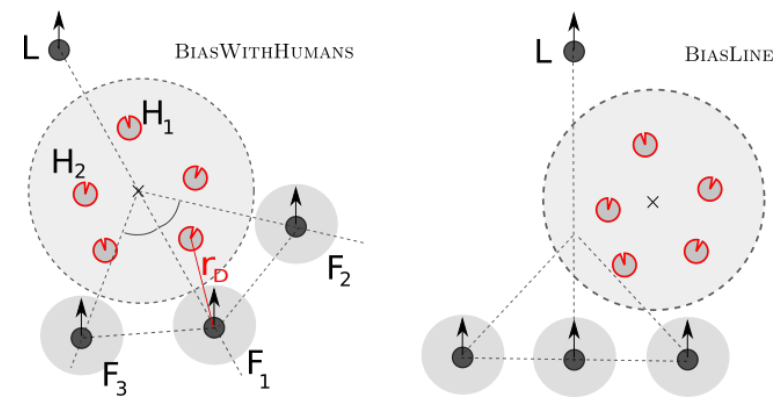

Fig. 1: The geometrical configuration of the formation is uniquely specified by the bias, which is a function of desirable human-robot distance $r_{D} . L$ denotes the Leader, $F$ denotes a Follower and $H$ a human Visitor. BiasWithHumans (left) and BIASLINE (right).

\section{Norm Realization: From Norms to Behaviors}

Norms stated for abstract entities in $\mathcal{I}$ must hold for the corresponding concrete entities in $\mathcal{D}$. Translation of norms is dependent on the domain [3], i.e. each norm defined over Roles, Acts, Arts is realized over $A, B, O$, which are the elements of the physical system. By illustrating with an example how abstract norms can be translated in terms of low-level parameters of complex behaviors, we will define the steps needed for bridging the abstraction-to-implementation gap of the normative systems [3].

\section{A. Illustrative Examples}

Consider a formation of $m$ humans and $n$ robots shown in Figure 1. Each robot in the formation is assigned a unique role of Leader $L$, or Follower in $F=\left\{F_{1}, \ldots, F_{n-1}\right\}$. The Leader robot guides the formation through the environment. The Follower robots maintain a rigid formation with each other and with the Leader based on a bias $\mathbf{b} \in \mathbb{R}^{n \times n \times 2}$ that uniquely defines a geometrical configuration in two dimensions. Humans with the roles of the Visitors $V=\left\{V_{1}, \ldots, V_{m}\right\}$ are uncontrollable agents. In general, humans follow instructions of following behind the Leader, and when not, we assume that human behavior can be influenced by the robots. Since human motion is similar to loose flocking rather than to rigid formation, Followers incorporate humans by generating repulsive fields around them. The examples below concern the Follower robots performing a behavior called MovelnFormation.

The first norm $n_{1}$, specified in human language (left column of the table below) can be realized as follows (right column):

\begin{tabular}{|c|c|}
\hline Norm $n_{1}$ & Realization \\
\hline \multirow{4}{*}{$\begin{array}{l}\text { When guiding } \\
\text { humans in a } \\
\text { formation } \\
\text { the followers } \\
\text { should keep a } \\
\text { configuration } \\
\text { so as to keep } \\
\text { the humans be- } \\
\text { hind the leader. }\end{array}$} & $\begin{array}{l}\text { The norm is active when humans are } \\
\text { known to be following behind the Leader } \\
\text { and Leader position is known }\end{array}$ \\
\hline & (Role assignment) \\
\hline & $\begin{array}{l}\text { The norm determines geometry of the robot } \\
\text { formation, i.e. the bias parameter }\end{array}$ \\
\hline & $\begin{array}{l}\text { Followers should attain a configuration sim- } \\
\text { ilar to Figure 1: desirable distance } r_{D} \\
\text { between the Followers and the humans is a } \\
\text { parameter of the configuration. }\end{array}$ \\
\hline
\end{tabular}

Furthermore, to respect $n_{1}$, the Follower robots use the calculated value of the bias in the MovelnFormation behavior, to drive towards the desired geometrical configuration. The $n_{1}$ norm is satisfied when the Follower robots are close to the desired places in the formation.

The realization of a second norm $n_{2}$ is as follows:

\begin{tabular}{|c|c|}
\hline Norm $n_{2}$ & Realization \\
\hline \multirow{4}{*}{$\begin{array}{l}\text { When guiding hu- } \\
\text { mans in a formation } \\
\text { the followers } \\
\text { should keep a com- } \\
\text { fortable distance } \\
\text { (distance) from the } \\
\text { humans. }\end{array}$} & $\begin{array}{l}\text { The norm is active only when humans } \\
\text { follow behind the Leader }\end{array}$ \\
\hline & $($ role $)$ \\
\hline & $\begin{array}{l}\text { The distance is assured by the robot } \\
\text { creating a repulsive field }\end{array}$ \\
\hline & $\begin{array}{l}\text { Weight of the repulsive field is a } \\
\text { function of the human-robot distance. }\end{array}$ \\
\hline
\end{tabular}

To respect $n_{2}$, a robot dynamically creates a repulsive field around the human to prevent interference with human social spaces when moving in a formation. The norm is satisfied when the human-robot distance is more than a threshold.

\section{B. Summary}

A) A norm is active if all its required conditions are satisfied

B) Each active norm $n_{i}$ can activate/modify/disable a subset of behavioral parameters

C) Parameters attain values that depend on state variables $R$ and knowledge $K$ (e.g., based on human models).

D) Behaviors can be readily and directly deployed with the given parameter values

E) A norm is satisfied if its outcome conditions are satisfied

\section{Parameters and Values}

Norm realization sets the parameters of the behaviors with the selected parameter values. An institution $I \in \mathcal{I}$ with norms Norms $=\left\{n_{1}, n_{2}, \ldots\right\}$, regulates behaviors in $B=\left\{b_{1}, b_{2}, \ldots\right\}$ of agents in $A$. Behaviors are parametric, i.e. they have multiple modalities $\gamma=b\left(p \in P, v_{p} \in V_{p}\right)$ that depend on a number of parameters $P$ and their values $V_{p}$. In general, $P$ is an index set of parameters, where for each $p \in P$ we associate a set of values $V_{p}$ from a family of sets $V(P)=\left\{V_{p}\right\}_{p \in P}$. The norm realization, by setting behavior parametrization to concrete and discrete values, constraints the choice of all possible behavior modalities to only one viable option.

As an example, consider the behavior Movelnteract that has two parameters, speed with the possible values $V_{\text {speed }} \in\{0,0.3,0.5\} \mathrm{ms}^{-1}$ and color of lights with values $V_{\text {lights }} \in\{$ green, blue $\}$. Then the set of all possible values of the set of parameters $P=\{$ speed, lights $\}$ is $V(P)=V_{\text {speed }} \times V_{\text {lights }}$, where for each $p \in P, v_{p}$ is a concrete value of $p$, e.g., $v_{p}=$ green $\in V_{\text {lights. }}$. The set of behavior modalities is $\gamma=\operatorname{Movelnteract}\left(p \in P, v_{p} \in V_{p}\right)=\left\{\left(p, v_{p}\right) \mid p \in P, v_{p} \in V_{p}\right\}$.

\section{Rules}

Rules are the binding material connecting norms to behaviors. In a one-to-one relation with the summary listed in Section III-B, we distinguish the following rules.
A) Requirement Rules
$r^{N}: 2^{C} \rightarrow \mathcal{N}$
B) Choice Rules
$r^{P}: \mathcal{N} \times B \rightarrow 2^{P}$
C) Value Rules
$r^{V}: P \times 2^{K} \times 2^{R} \rightarrow V$
D) Application Rules
$r^{B}: 2^{P} \times 2^{V} \rightarrow \Gamma$
E) Outcome Rules
$r^{O}: \mathcal{N} \times \mathcal{W} \rightarrow \mathcal{N}^{\mathcal{T}}$

where $\mathcal{N}$ is the set of active norms, $\mathcal{N}^{\mathcal{T}}$ is the set of satisfied norms and $\Gamma$ is the set of behavior modalities. Rules $r^{N}$ 


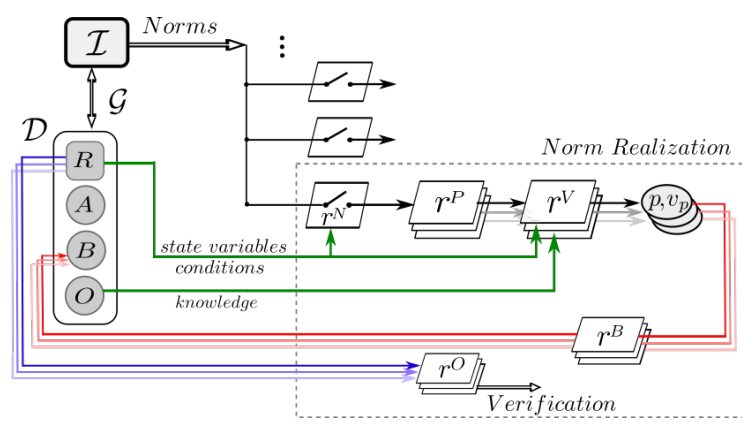

Fig. 2: Norm realization $\mathcal{K}$ translates abstract norms into constraints over behaviors modalities by setting behavior parameters $P$ to concrete values $V_{p}$ using a set of rules.

activate norms based on conditions $C, r^{P}$ give a choice of parameters that should be altered for the given behavior, $r^{V}$ provide recipes to set the parameter values based on knowledge $K$ and state variables $R, r^{B}$ enact behavior procedures for applying parameter values and $r^{O}$ verify norm compliance after the behavior changes the world state.

A given physical system $\mathcal{D}$ behaves in compliance with an institution $\mathcal{I}$ if for each norm $n \in \mathcal{N}_{t}$ at every time $t$, the world state $\mathcal{W}_{t}$ remains within the boundaries $\mathcal{W}_{t} \in \mathcal{W}^{\star}$ :

$$
\mathcal{W}^{\star}=\{s \mid f(s)=\text { True }, s \in \mathcal{S}\},
$$

where $f(s)$ is a function that is True when $s$ is inside the bounds defined by $r_{s}^{O}$. The relations between the rules and the other elements of the domain are shown in Figure 2.

Rules operate on formulas that can be applied directly to the robot behaviors and therefore they provide the norm realization for the norms that are inherently vague [3].

Definition 6: The norm realization is a tuple

$$
\mathcal{K}=\left\langle r^{N}, r^{P}, r^{V}, r^{B}, r^{O}\right\rangle
$$

Norm realization can be readily applied in a robotic language. In Algorithm 1 we show one possible approach for implementing norm realization, and we use this method for the remainder of the paper. For a norm $n_{k}$, rule $r_{k}^{N}$ evaluates a set of conditions $c_{k}$, rule $r_{k}^{P}$ returns a set of parameters $p_{k}$, rule $r_{k}^{V}$, given knowledge $K_{t}$ and state variables $R_{t}$, returns value $v_{p}$ of a parameter $p \in p_{k}$. Rule $r_{b}^{B}$ enacts a procedure for applying parameter $p$ with its value directly on the behavior $b$ and so, it has to be implemented as part of the behavior. Finally, outcome rule $r_{k}^{O}$ checks if the desired bounds for states in $\mathcal{W}_{t+1}$ are satisfied.

\section{CAse Study}

The goal of this case study is to show how a physical system with robots and humans can be governed using institutions. We demonstrate that the norm realization allows for introducing normative dimension in complex, collective behaviors of heterogenous agents. Our case study consists of two phases. In the first phase, a group of humans is guided by a number of robots to a large room. The formation includes one Leader, human Visitors, and Followers. The second phase takes place in the room, where two cooperative agents take the Visitors on a tour. One of the agents, the Tutor, describes the objects, while the other agent, the Assistant, moves around the classroom, showing the objects to the Visitors.

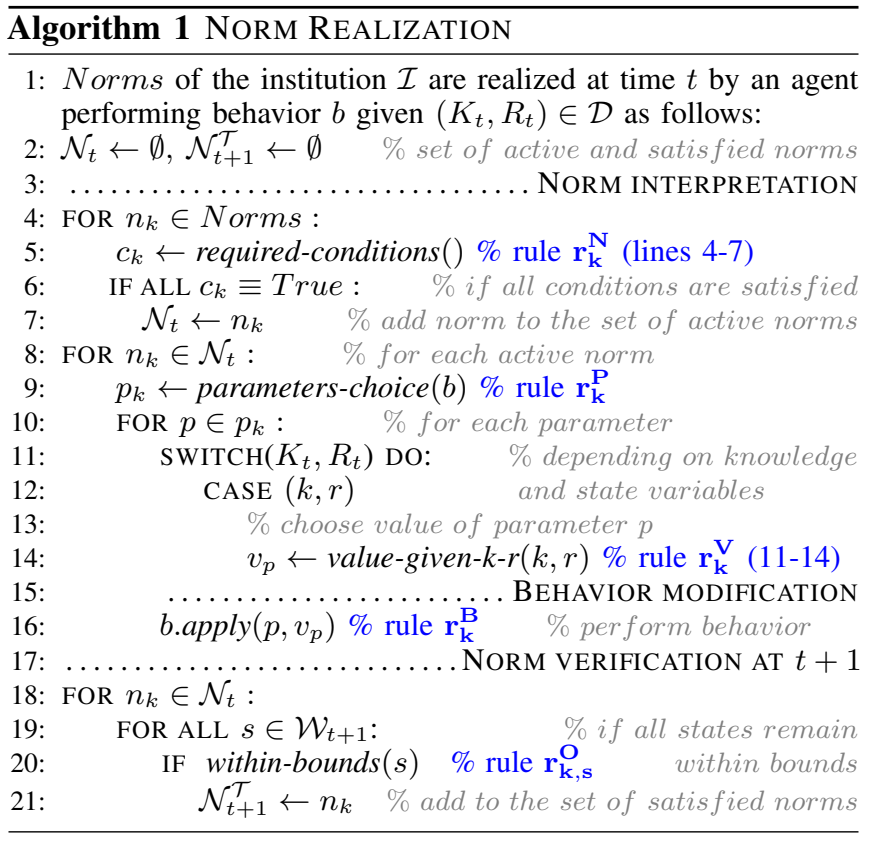

The case study is governed by two institutions. The Guidance institution in phase one, and the Tutoring institution in phase two. The Guidance institution is defined as follows:

$$
\begin{aligned}
\text { Guidance } & =\left\langle\text { Arts }_{G}, \text { Roles }_{G}, \text { Acts }_{G}, \text { Norms }_{G}\right\rangle \\
\text { Roles }_{G} & =\{\text { Leader, Follower, Visitor }\} \\
\text { Acts }_{G} & =\{\text { Guide, AssistGuidance }\} \\
\text { Arts }_{G} & =\{\text { EntranceHall, Proxemics, SocialForces }\} \text {. }
\end{aligned}
$$

with four social norms $\operatorname{Norms}_{G}=\left\{n_{1}, n_{2}, n_{3}, n_{4}\right\}$, where $n_{1}$ and $n_{2}$ are given as examples in Section III-A. Norm $n_{3}$ states "When humans fail to follow behind the leader during guidance, the leader should wait for them" and $n_{4}$ "When humans guided in a formation are not following the leader, the followers should encourage them to return". In this work we focus on the methods for introducing normative aspect to the behaviors and do not provide the full formal description of the case study. For additional information please refer to [25].

The description so far provides specification for an abstract institution. We now ground our institutions in the domain of the case study involving real robots and humans. The domain includes the following components (only components to which the Guidance institution is grounded are listed):

$$
\begin{aligned}
& A=\left\{\text { mbot }_{1}, \text { mbot }_{2}, \text { participant }_{1}, \text { participant }_{2}\right\} \\
& B=\{\text { MoveOnTrajectory }, \text { MovelnFormation }\} \\
& O=\left\{\text { corridor }_{\text {proxemic }}, \text { force }_{\mathrm{x}}\right\}
\end{aligned}
$$

where we use the subscript $(\cdot)_{x}$ to abbreviate several affordances that involve agent/object $(\cdot)$. Knowledge $K$ of the domain includes proxemic $x_{x}=\left\{\right.$ social $_{x}$, intrusive $\left.e_{x}\right\}$ and force . $_{x}$ For example, if the types of agents are given as state variables agent $_{\mathrm{x}}$. type $\in R$, where $\operatorname{vals(type)} \in\{$ robot, adult, child $\}$, we can specify that the space where the average child may feel disconcerted is expected to be around $1.2 \mathrm{~m}$ using knowledge intrusive $_{\text {child }}=1.2 \mathrm{~m}$. Similarly, we can encode preferences known from social studies (e.g., Proxemics, Social Forces), or preferences of individuals learned experimentally. The 
Guidance institution is grounded with $\mathcal{G}_{\text {Guidance }}$ :

$\mathcal{G}_{A}=\left\{\left(\right.\right.$ Leader mbot $\left._{1}\right),\left(\right.$ Follower, mbot $\left._{2}\right),\left(\right.$ Visitor, participant $\left.\left.{ }_{\mathrm{x}}\right)\right\}$

$\mathcal{G}_{B}=\{($ Guide, MoveOnTrajectory $)$, (AssistGuidance, MovelnFormation $\left.)\right\}$

Artifact grounding $\mathcal{G}_{O}$ includes (EntranceHall, corridor) pair as well as the grounding of knowledge. For the norms used in our case study, we use the following knowledge:

$$
\begin{aligned}
K=\{ & \text { social }_{\text {child }}: r_{D}=2.0 \mathrm{~m}, \text { social }_{\text {adult }}: r_{D}=1.6 \mathrm{~m}, \\
& \text { intrusive }_{\text {child }}: r_{D}=1.2 \mathrm{~m}, \mathrm{intrusive} \mathrm{e}_{\text {adult }}: r_{D}=1.0 \mathrm{~m}, \\
& \text { force }_{\text {child }}:\left(K_{0}=1.2, \Delta_{C}=0.65, \Delta_{A}=2.2\right), \\
& \text { force } \left._{\text {adult }}:\left(K_{0}=1.0, \Delta_{C}=0.55, \Delta_{A}=1.6\right)\right\}
\end{aligned}
$$

where $K_{0}, \Delta_{C}$ and $\Delta_{A}$ are defined in Section IV-A.2.

\section{A. Normative Robot Behaviors}

Agents in the realized system take the institutional roles with the associated actions that are grounded to behaviors. We assume that an agent can take a role only if it can carry out the grounded behavior. Therefore a Leader can perform a MoveOnTrajectory behavior, a Follower the MovelnFormation behavior, and a Tutor and an Assistant of the Tutoring institution can perform the PerformDialog and ShowObject behaviors, respectively. In this section we describe the behaviors that are available to the robot agents. Human behaviors are not represented formally.

1) MoveOnTrajectory: The task of the Leader is to plan a path in a known environment and guide the team along this path, while making its current position known to all the agents in the team. The Leader robot plans the path using a Fast Marching Method (FMM). Given a goal location, FMM is used to obtain a local-minima-free potential field encoding the optimal direction of motion towards the goal, for any given point, provided that the goal is reachable from that point. The Leader independently follows the planned path with a desired speed, providing trajectory for the team members.

2) MoveInFormation: The task of a Follower is to assist the Leader in guiding the Visitors. Each Follower robot maintains desired distances with respect to the Leader and with respect to the other Follower robots. All robots assigned the Follower role act following a Laplacian-based consensus law, which drives them to the desired places in the formation [27]. The collection of the desired inter-robot distances forms a bias that fully defines a rigid geometrical configuration of the robot formation. A state variable pose of an agent $i$ is denoted $\mathbf{x}_{i}=\left(x_{i}, y_{i}, \alpha_{i}\right)$ and position $\overline{\mathbf{x}}_{i}$. Each $F_{i}$, achieves the formation using:

$$
\dot{\overline{\mathbf{x}}}_{i}=\frac{1}{\left|\sum_{j} \mathcal{L}_{i j}\right|} \sum_{i \sim j}-\mathcal{L}_{i}\left[\left(\overline{\mathbf{x}}_{i}-\overline{\mathbf{x}}_{j}\right)-b_{i j}\right]
$$

where $\mathcal{L}$ is a non-stationary Laplacian, $\mathbf{b}_{i} \in \mathbb{R}^{\Delta \times 2}$ is the bias of $F_{i}$ and $i \sim j$ means that robots $i$ and $j$ are connected (they communicate). In this work, poses of all the agents in the formation are shared. Finally, we assume that the Followers are omni-directional, so the heading is controlled so as to match the orientation of the Leader $\alpha_{L}, \dot{\alpha}_{i}=\alpha_{L}-\alpha_{i}$. Details of the formation control algorithm can be found in [27].

The Visitors perform independent behavior, and as such, they do not conform to the rigid configuration specified by

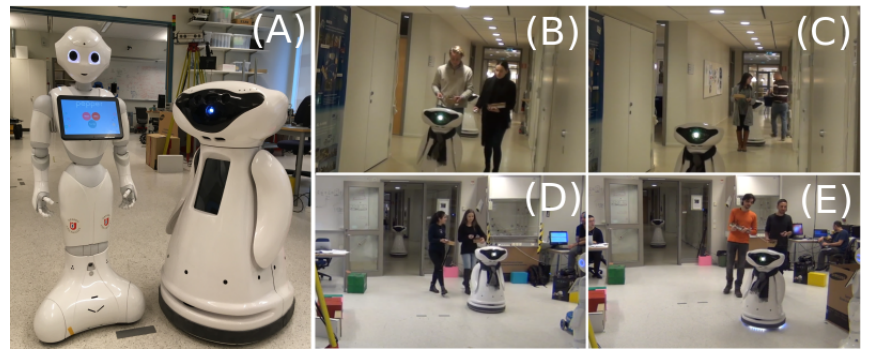

Fig. 3: (A) Robots participating in the experiments, the MBot robot (right) and Pepper robot (left). Snapshots of the experiments during phase one (B-C) and phase two (DE), for Scenario I (B,D,E) and Scenario II (C), at at the time when the participant stopped following behind the Leader.

the bias. They are included in the formation as follows: each Visitor $V_{h}$ generates a repulsive field that modifies the motion of $F_{i}$ as follows:

$$
\dot{\overline{\mathbf{x}}}_{i}=\dot{\overline{\mathbf{x}}}_{i}+\sum_{i \sim h} w_{i h}\left(\left\|\overline{\mathbf{x}}_{h}-\overline{\mathbf{x}}_{i}\right\|\right) \cdot\left(\overline{\mathbf{x}}_{h}-\overline{\mathbf{x}}_{i}\right)
$$

where $i \sim h$ means that $i$ receives state information of $h$. The weight coefficient $w_{i h}$ that depends on distance $d=\left\|\overline{\mathbf{x}}_{h}-\overline{\mathbf{x}}_{i}\right\|$ has a repulsive effect, assuring that $F_{i}$ stays away from $V_{h}$ :

$$
w_{i k}= \begin{cases}-\frac{K_{0}}{\left(d-\Delta_{c}\right)^{2}}-\frac{K_{0}}{\left(\Delta_{a}-\Delta_{c}\right)^{2}} & \text { if } \Delta_{c}+\epsilon_{c}<d<\Delta_{a} \\ -\frac{K_{0}}{\epsilon_{c}^{2}}-\frac{K_{0}}{\left(\Delta_{a}-\Delta_{c}\right)^{2}} & \text { if } 0 \leq d<\Delta_{c}+\epsilon_{c} \\ 0 & \text { otherwise }\end{cases}
$$

The weight changes continuously and is parametrized with constant gain $K_{o}, \Delta_{a}$, the range where repulsion is quadratic function of $d$, and $\Delta_{c}<\Delta_{a}$, an imminent collision range, where the weight grows to a large, finite number. A collection of weights $\mathcal{C}_{i}=\left\{w_{i h} \mid(i \sim h)\right\}$ is the parameter of the formation.

3) Tutoring Behaviors: The Tutor and the Assistant synchronize execution of their behaviors. The Tutor describes an object $o_{i}$ with the PerformDialog $\left(o_{i}\right)$ behavior, while Assistant navigates to that object with the behavior ShowObject $\left(o_{i}\right)$.

\section{B. Realization of Norms}

We provide a realization of norms $n_{1}, n_{2} \in$ Norms $_{G}$ from Section III-A. The realization $\mathcal{K}_{1}=\left\langle r^{N}, r^{P}, r^{V}, r^{B}, r^{O}\right\rangle$ is:

$$
\begin{aligned}
& \mathcal{N}_{t}=r^{N}\left(c_{1}\right)=\left\{\begin{array}{l}
n_{1} \in \mathcal{N}_{t} \text { if } c_{1}=\text { True } \\
n_{1} \notin \mathcal{N}_{t} \text { otherwise }
\end{array}\right. \\
& p_{1}=r^{P}\left(\mathcal{N}_{t}, \text { MovelnFormation }\right)=\left\{\mathbf{b}_{i}\right\} \\
& v_{p_{1}}^{j}=r_{j}^{V}\left(p_{1}, k_{h}, r_{h}\right)=f_{\mathbf{b}}\left(r_{D}\right) \text { with }\left\{\begin{array}{l}
r_{D}=1.6 \text { if adult } \in r_{h}, \text { social } \in k_{h} \\
r_{D}=2.0 \text { if child } \in r_{h}, \text { social } \in k_{h}
\end{array}\right. \\
& \gamma=r^{B}\left(p_{1}, v_{p_{1}}\right)=\text { MovelnFormation. apply }\left(p_{1}, v_{p_{1}}\right) \\
& \mathcal{N}_{t+1}^{\mathcal{T}}=r^{O}\left(\mathcal{N}_{t}, \mathcal{W}_{t+1}\right)=\left\{\begin{array}{l}
n_{1} \in \mathcal{N}_{t+1}^{\mathcal{T}}, \text { if } e_{F}-e_{F}^{M A X} \leq 0 \\
n_{1} \notin \mathcal{N}_{t+1}^{\mathcal{T}}, \text { otherwise }
\end{array}\right.
\end{aligned}
$$

where $c_{1}=$ \{LEADER_POSE_KNOWN, VISITORS_FOLLOW_LEADER $\}$, $e_{F}$ is formation error and $h$ denotes a visitor, and so, $r_{h}$ and $k_{h}$ are the state variables and the knowledge that regard visitor $h$. The method $f_{\mathbf{b}}\left(r_{D}\right)$ finds the exact value for the matrix $\mathbf{b}_{i}$ given $r_{D}$. When the positions of Visitors are available in $R$, the bias defines shape similar to Figure 1 (left) (i.e. 


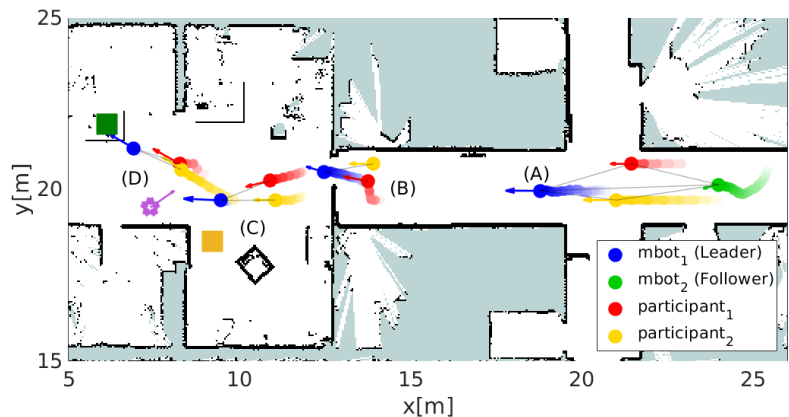

Fig. 4: Trajectories of the robots and the participants in Scenario I, shown for the times $t=11 s$ (A), $t=35 s$ (B), $t=59 s(\mathrm{C})$ and $t=72 s(\mathrm{D})$.

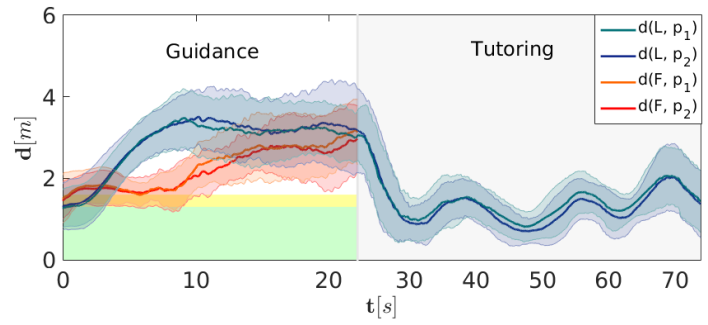

Fig. 5: Average distances for Scenario I. Yellow area is when $n_{2}$ is not satisfied $\left(d\left(F, p_{x}\right)\right.$ below $\left.\left.\Delta_{a}=1.6 \mathrm{~m}\right)\right)$.

BiasWithHumans). This bias is calculated dynamically, so that $F_{i}$ keeps a distance from $L$ that places the desired position of $F_{i}$ behind all $H_{h} \in H$. For a single human guidance, BiasWithHumans is chosen so as the desired robot-human distance is $2 r_{D}$. When more than one humans are guided, the bias regulates the desired robot-human distance to $r_{D}$. When human positions are not available (e.g., due to localization system failure), the method returns a default BIASLine, the calculation of which is independent of the positions of humans (see Fig. 1, right). In previous work [27], we have described how to change the local bias of a robot. With the new bias the robot drives to the desired place in the formation (see Eq. 1). The realization of norm $n_{2}$ for $\operatorname{robot} F_{i}$ is $\mathcal{K}_{2}=\left\langle r^{N}, r^{P}, r^{V}, r^{B}, r^{O}\right\rangle$ with:

$\mathcal{N}_{t}=r^{N}\left(c_{2}\right)=\left\{\begin{array}{l}n_{2} \in \mathcal{N}_{t} \text { if } c_{2}=\text { True } \\ n_{2} \notin \mathcal{N}_{t} \text { otherwise }\end{array}\right.$

$p_{2}=r^{P}\left(\mathcal{N}_{t}\right.$, MovelnFormation $)=\left\{\mathcal{C}_{i}\right\}$

$v_{p_{2}}^{j}=r_{j}^{V}\left(p_{2}, k_{h}, r_{h}\right)=f_{\mathcal{C}}\left(K, \Delta_{C}, \Delta_{A}\right)$ with

$\left\{\begin{array}{l}K_{0}=1.0, \Delta_{C}=0.55, \Delta_{A}=1.6 \text { if adult } \in r_{h}, \text { social } \in k_{h} \\ K_{0}=1.2, \Delta_{C}=0.65, \Delta_{A}=2.2 \text { if child } \in r_{h}, \text { social } \in k_{h}\end{array}\right.$
$\gamma=r^{B}\left(p_{2}, v_{p_{2}}\right)=$ MovelnFormation. apply $\left(p_{2}, v_{p_{2}}\right)$

where $c_{2}=\{$ VISITORS_POSITIONS_KNOWN, VISITORS_FOLLOW $\}$ and $f_{\mathcal{C}}\left(K, \Delta_{C}, \Delta_{A}\right)$ calculates the weight $w_{i v} \in \mathcal{C}_{i}$ using Eq. 3 and modifies motion of $F_{i}$ according to Eq. 2, obliging $F_{i}$ to keep away from the personal space of $H_{h}$.

Norms $n_{3}$ and $n_{4}$ are active given the conditions \{VISITORS_POSITIONS_KNOWN, NOT VISITORS_FOLLOW\}. Realization $\mathcal{K}_{3}$ sets the desired speed parameter of the MoveOnTrajectory behavior carried out by the Leader robot to zero, for the duration when the norm is active. Norm is satisfied when $d\left(L_{t}, L_{t_{0}}\right) \leq D_{L}$, where $D_{L} \rightarrow 0$ is a threshold on maximal distance $L$ is allowed to move away after the norm becomes active. Finally, realization $\mathcal{K}_{4}$, similarly as $\mathcal{K}_{1}$, changes $\mathbf{b}_{i}$ based on $r_{D}$, as defined by intrusive child and intrusive $_{\text {adult }}$ in $K$. Smaller values of intrusive spaces compared to social bring $F_{i}$ closer to $V_{v}$, with the intent of encouraging visitor's return to the team. Norm $n_{4}$ is evaluated with $e_{F}$.

The formation error $e_{F}=\left(\left|\sum_{j} \mathcal{L}_{i j}\right|\right)^{-1} \sum_{i \sim j}\left|\left(\overline{\mathbf{x}}_{i}-\overline{\mathbf{x}}_{j}\right)-b_{i j}\right|$ is the average difference between the desired distances and the actual distances between the agents in the formation. A distance $\left\|\overline{\mathbf{x}}_{a}-\overline{\mathbf{x}}_{b}\right\|$ is denoted $d(a, b)$.

\section{EXPERIMENTS}

Experiments have been performed using holonomic MBot robots $^{1}$ and a Pepper robot $^{2}$ (Fig. 3). The MBot robots self-localize using $\mathrm{AMCL}^{3}$ provided in ROS. All robots connect to a local wireless network. Robot behaviors are distributed, behaviors are activated by a central planner. Human localization is achieved with Kio Ultra-wide band technology from Eliko ${ }^{4}$. Videos accompanying this paper can be found at: https://disal.epfl.ch/research/ InstitutionalRoboticsFormations.

\section{A. Scenarios}

We distinguish two scenarios with the aim of demonstrating the effect of introducing normative behaviors in real environments with human participants. They both consist of a Guidance and a Tutoring phase, Scenario I involves two human Visitors. During the Guidance phase Visitors are asked to follow behind the Leader robot (for distinction, wearing a scarf). A single Follower robot accompanies the participants, while attempting to act in accordance with the norms $n_{1}$ and $n_{2}$. After the Leader enters the next room, thus beginning the Tutoring phase, the Follower moves aside and the Leader robot assumes the role of an Assistant for a third robot serving as Tutor. The Tutor robot describes objects present in the scene, while the Assistant robot "shows" those objects by moving close to them. Scenario II is essentially similar to Scenario I but involves only one human Visitor, who is asked to stop following the Leader for around $5 s$ during the Guidance phase of the experiment.

We chose $e_{F}^{M A X}=0.65 \mathrm{~m}$, equal to the diameter the MBot robot. Condition VISITORS_FOLLOW_LEADER is evaluated to true when $\forall H_{h} \in$ Visitors, $d\left(L, H_{h}\right) \leq D_{v}$, where $D_{v}=\infty$ for Scenario I (so that $n_{1}$ and $n_{2}$ are always active) and $D_{v}=3.2 \mathrm{~m}$ for Scenario II. For safety reasons, we limited the maximum velocity of $F$ robots along forward and sideway axes to $v_{\max }=1 \mathrm{~ms}^{-1}$. Values $r_{D}, K_{o}, \Delta_{C}$ and $\Delta_{A}$ of knowledge $K$ were obtained experimentally in [25], according to the Social Forces model [12] of human motion.

1) User Study: For Scenario I, we performed 20 experiments with altogether 40 participants, ages $19-46$, recruited among researchers and students with various backgrounds. Half of the group has been informed about the course of the events, including explanation of robot roles and their

\footnotetext{
${ }^{1}$ Developed during Multi-Robot Cognitive Systems Operating in Hospitals (MOnarCH), FP7, FP7-ICT-2011-9-601033 (http://monarch-fp7.eu)

${ }^{2}$ SoftBank Robotics https://www.ald.softbankrobotics.com

${ }^{3}$ Adaptive Monte Carlo Localization (http://wiki.ros.org/amcl)

${ }^{4}$ Eliko https://www.eliko.ee
} 


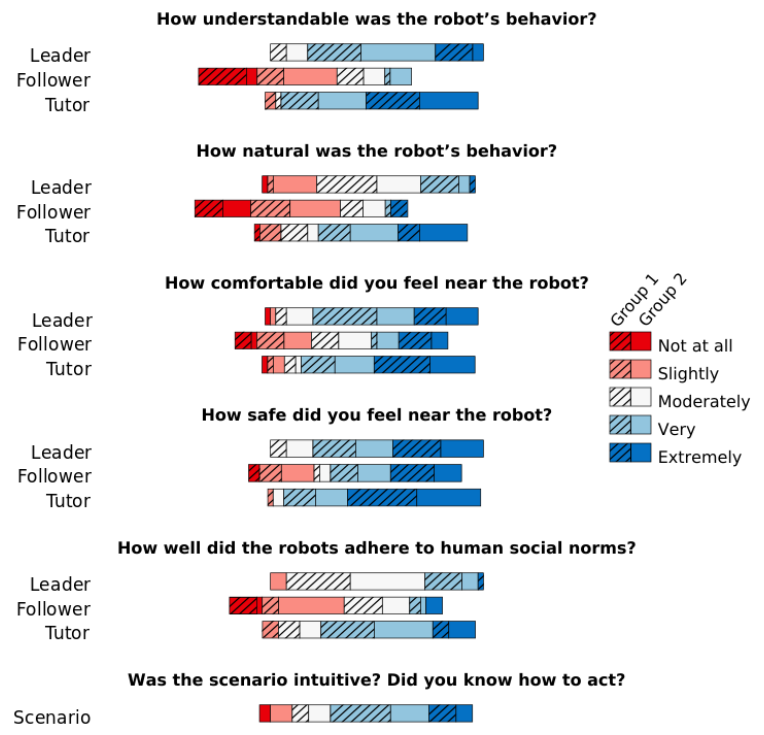

Fig. 6: Results of the user-based evaluation on five-level Likert scale. Group 1 involved uninformed participants and Group 2 informed users.

behaviors, while the other half was only given the instruction of following the Leader. After the experiments we handed out questionnaires with questions listed in Figure 6, evaluated on five-level Likert scale. For Scenario II we performed 10 experiments with 10 participants. Pictures taken during the experiments are shown in Figure 3.

2) Temporal Planning: The temporal course of the experiments is established by an offline plan devised by a temporal planner [25]. The planner determines relations between time intervals when the behaviors execute, based on institution specification, domain, semantics, and grounding, which are used to automatically generate a planning domain [26]. The planner enforces the temporal norms, while the norm realization enforces the spatial norms.

\section{B. Analysis of Results}

1) Scenario I: Execution of Scenario I is illustrated in Figure 4. It starts with the robots mbot $_{1}$ grounded to Leader role and mbot $_{2}$ grounded as Follower, guiding two adult Visitors, participant ${ }_{1}$ and participant ${ }_{2}$ through a corridor (A), to the entrance of the room (B), when the institution becomes dormant. At this point, the Tutoring institution becomes active, with mbot $_{1}$, grounded to the Assistant role, and pepper, grounded to the Tutor role. Two objects are chosen from the set $\{$ VR_SET, ROBOT_STATION, CAMERA $\}$, and presented by the robots to the Visitors (C and D).

The norms $n_{1}$ and $n_{2}$ are active throughout the MovelnFormation behavior of $\operatorname{mbot}_{2}(t=0-24 s)$ because the condition VISITORS_FOLLOW is always true. On average, after initialization $(t=0-3 s)$, the semantics of $n_{1}$ are satisfied for around $5 s$ with $e_{F} \leq 0.65 \mathrm{~m}$. After this, at $t=8 s$ on average, $e_{F}$ increases and stabilizes at around $1.5 \mathrm{~m}$. Log data suggests that the increase of $e_{F}$ is caused by saturation of the speed of mbot $_{2}$ to $v_{\max }$, illustrating the tradeoff between safety and performance. The distances used for evaluating $n_{2}, d\left(\mathrm{~F}, p_{x}\right)$, where $p_{x}=\left\{p_{1}, p_{2}\right\}$ denotes the
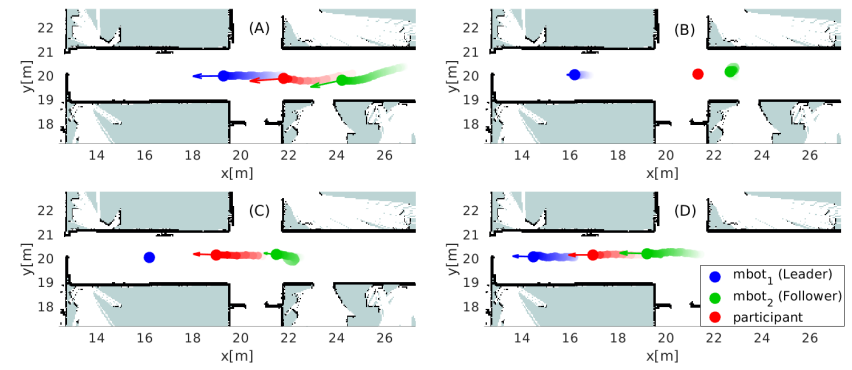

Fig. 7: Trajectories of the robots and the participants in Scenario II, shown for the times $t=16 s$ (A), $t=26 s$ (B), $t=34 s(\mathrm{C})$ and $t=39 \mathrm{~s}$ (D).

participants, are shown in Figure 5. On average, the distances preserve the set point of $\Delta_{a}=1.6 \mathrm{~m}$, and $n_{2}$ is satisfied.

Results of the survey, presented in Figure 6 show that in general, the Follower was perceived as the least understandable and natural among the robots. Moreover, the participants did not observe that the Follower robot was adhering to social norms. The Tutor robot was given the highest scores. Whether or not the participants were given a priori expectation of the experiment did not have a significant effect on the perception of the robots. Among the most notable differences between the groups we noted that the informed group judged the Leader as slightly less understandable, less natural and less comfortable to be around. On the other hand, the same group understood the role of the Follower better. Results suggest that the acceptance does not depend on whether the behavior of the robot is explained. It is possible that the behavior of a robot is perceived on a subconscious level, and explaining the behavior has little effect on perception of robot friendliness.

2) Scenario II: Snapshots of representative experimental run are shown in Figure 7. After the team attains initial steady-state at $t=16 \mathrm{~s}$ (top left), the participant stops (top right at $t=26 \mathrm{~s})$. When $d(L, p) \geq D_{v}=3.2 \mathrm{~m}$, the condition V_FOLLOW_L becomes false, which causes activation of $n_{3}$ and $n_{4}$. Due to $n_{3}$, the Leader sets its speed to zero and due to $n_{4}$, the Follower robot reduces the bias according to the proxemics intrusive adult . After the participant re-joins the Leader, as determined by $d(L, p) \leq D_{v}$ (bottom left, $t=34 s$ ), the norms $n_{3}$ and $n_{4}$ are no longer active and the team attains a steady state defined by $n_{1}$ and $n_{2}$ (bottom right, $t=39 \mathrm{~s}$ ).

How the value of $d(L, p)$ drives the behaviors of the robots through the norms can be further analyzed in Figure 8, which shows correlation between $d(L, p)$ and $d(F, p)$. According to $n_{1}$, BiasWithHumans regulates the robothuman distance to $2 r_{D}$ for a single human guidance, so for $d(L, p) \leq D_{v}, d(F, p)$ tends to $3.2 m$ (blue region in Figure 8). For $d(L, p) \geq D_{v}, d(F, p)$ (red region) tends to the value $r_{D}=1.0 \mathrm{~m}$ given by intrusive adult , and enforced by $n_{4}$.

\section{Discussion}

Norms of human societies are not always suitable to be directly applied to robots, or can be applied in some situations but not others. In our example, the Follower robot, being the only agent that explicitly acted according to social rules, was not perceived as more social than the other robots. Some results reported in the literature are in accordance with our findings [14], [15], while some convey the opposite [28]. 


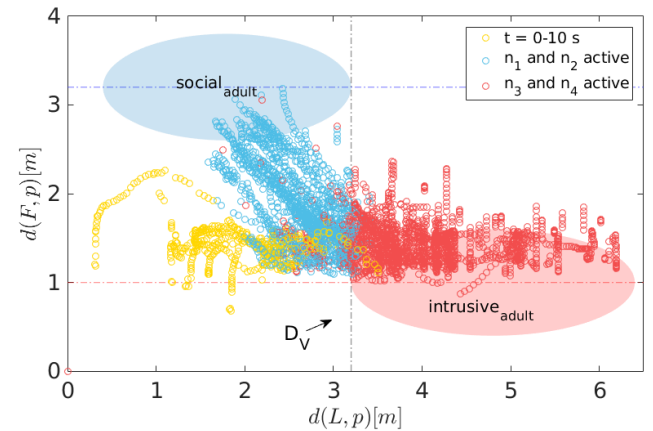

Fig. 8: Scenario II. Distances showing how norms shape behaviors of the Leader $(\mathrm{L})$ and the Follower $(\mathrm{F})$ robots. Blue region is the case when $n_{1}$ and $n_{2}$ are active, and value of social $_{\text {adult }}\left(2 r_{D}=3.2 \mathrm{~m}\right)$ dictates the Follower-human distance. After the participant (p) stops following, the red region is when Leader waits according to $n_{3}$, and Follower tends to value of intrusive adult $\left(r_{D}=1.0 \mathrm{~m}\right)$ given by $n_{4}$.

Results of our experiments evince that norms should always be verified against user expectations for the particular application.

Our user studies indicate that the chosen norms and their realizations are not well accepted by the users during the particular case of human guidance in formation. However, the fact that the norms are modeled explicitly allows us to focus of our future attention on design and re-design of norms, whereas thanks to norm realization, norms can be incorporated by the robots in a manner similar to plug-and-play principle.

\section{CONCLUSIONS}

To be accepted in human society, future robots will need to comply to the norms and conventions that humans use. Current trends hard-wire norms by implicitly programming norm-compliant robot behaviors. In this paper, we propose to encapsulate abstract norms into reusable structures, called institutions. Only then the institutions are grounded to concrete systems. We have shown that with norm realization, our approach can be used to introduce normative aspects into robot behaviors, thus allowing robots to participate in mixed human-robot societies that adhere to human-defined norms. During experiments carried out in real settings with human users we performed user-based evaluation aimed at studying user's acceptance of robots that follow human-defined norms. Our approach enables a systematic design of social norms to coordinate interaction between groups of robots and humans, which realization (introduced here) grounds the design to the application in real robots and real scenarios, further enabling its quantitative assessment through metrics such as the difference between the desired and actual behaviors (e.g., by comparing human-robot distances). The results suggest the future line of our research, which will focus on understanding how to generalize norm realization for spatiotemporal norms to be respected in human-populated environments.

\section{REFERENCES}

[1] G. Boella, L. Torre, and H. Verhagen, "Introduction to normative multiagent systems," Computational \& Mathem. Organization Theory, vol. 12, no. 2-3, pp. 71-79, 2006.

[2] E. Ostrom, Understanding Institutional Diversity. Princeton University Press, 2005.
[3] D. Grossi and F. Dignum, "From abstract to concrete norms in agent institutions," in Formal Approaches to Agent-Based Systems, pp. 12-29, Springer Berlin Heidelberg, 2005.

[4] G. Weiss, Multiagent Systems. The MIT Press, 2013.

[5] M. Esteva, D. De la Cruz, and C. Sierra, "ISLANDER: An electronic institutions editor," in Proc of the Int Joint Conf on Autonomous Agents and Multiagent Systems, pp. 1045-1052, 2002.

[6] M. Hannoun, O. Boissier, J. S. Sichman, and C. Sayettat, "MOISE: An organizational model for multi-agent systems," in Advances in Artificial Intelligence, pp. 156-165, 2000.

[7] M. Dignum, A model for organizational interaction: based on agents, founded in logic. PhD thesis, Utrecht University, NL, 2004.

[8] T. Kruse, A. K. Pandey, R. Alami, and A. Kirsch, "Human-aware robot navigation: A survey," Robotics and Autonomous Systems, vol. 61, no. 12, pp. $1726-1743,2013$.

[9] F. M. Carlucci, L. Nardi, L. Iocchi, and D. Nardi, "Explicit representation of social norms for social robots," in Proc of IEEE/RSJ Int Conf on Intelligent Robots and Systems, pp. 4191-4196, 2015.

[10] E. Ferrera, J. Capitán, A. R. Castano, and P. J. Marrón, "Decentralized safe conflict resolution for multiple robots in dense scenarios," Robotics and Autonomous Systems, vol. 91, pp. 179 - 193, 2017.

[11] E. T. Hall, The Hidden Dimension. Doubleday \& Co, 1966.

[12] D. Helbing and P. Molnár, "Social force model for pedestrian dynamics," Physical Review E, vol. 51, no. 5, pp. 4282-4286, 1995.

[13] B. Okal and K. O. Arras, "Formalizing normative robot behavior," in 8th Int Conf on Social Robotics, pp. 62-71, 2016.

[14] E. A. Sisbot, L. F. Marin-Urias, R. Alami, and T. Simeon, "A human aware mobile robot motion planner," IEEE Trans on Robotics, vol. 23, no. 5, pp. 874-883, 2007.

[15] G. Ferrer, A. G. Zulueta, F. H. Cotarelo, and A. Sanfeliu, "Robot social-aware navigation framework to accompany people walking sideby-side," Autonomous Robots, vol. 41, no. 4, pp. 775-793, 2017.

[16] R. Kirby, R. Simmons, and J. Forlizzi, "Companion: A constraintoptimizing method for person-acceptable navigation," in 18th IEEE Int Symposium on Robot and Human Interactive Communication, pp. 607612, 2009.

[17] C. P. Lam, C. T. Chou, K. H. Chiang, and L. C. Fu, "Human-centered robot navigation - towards a harmoniously human-robot coexisting environment," IEEE Trans on Robotics, vol. 27, no. 1, pp. 99-112, 2011.

[18] S. G. J.V. Gomez, Nikolaos Mavridis, "Social path planning: Generic human-robot interaction framework for robotic navigation tasks," in IROS workshop on Cognitive Robotics Systems: Replicating Human Actions and Activities, 2013.

[19] A. K. Pandey and R. Alami, "A framework towards a socially aware mobile robot motion in human-centered dynamic environment," in Proc of IEEE/RSJ Int Conf on Intelligent Robots and Systems, pp. 5855$5860,2010$.

[20] A. Wasik, A. Martinoli, and P. U. Lima, "An institutional robotics approach to the design of socially aware multi-robot behaviors," in Towards Intelligent Social Robots: Social Cognitive System in Smart Enviroments, 2017.

[21] D. C. North, "Institutions," Journal of Economic Perspectives, vol. 5, no. 1 , pp. 97-112, 1991.

[22] W. Kasper and M. E. Streit, Institutional Economics. No. 1582 in Books, Edward Elgar Publishing, 1999.

[23] P. Silva and P. U. Lima, "Institutional robotics," in Advances in Artificial Life: 9th European Conference on Advances in Artificial Life, pp. 595604, 2007.

[24] J. N. Pereira, P. Silva, P. U. Lima, and A. Martinoli, "Formalization, Implementation, and Modeling of Institutional Controllers for Distributed Robotic Systems," Artificial Life, vol. 20, no. 1, pp. 127-141, 2013.

[25] S. Tomic, A. Wasik, P. U. Lima, A. Martinoli, F. Pecora, and A. Safiotti, "Towards institutions for mixed human-robot societies," in Int Joint Conf on Autonomous Agents and Multiagent Systems, pp. 2216-2217, 2018.

[26] S. Tomic, F. Pecora, and A. Saffiotti, "Norms, institutions, and robots," arXiv preprint arXiv:1807.11456, 2018.

[27] A. Wasik, J. N. Pereira, R. Ventura, P. U. Lima, and A. Martinoli, "Graph-based distributed control for adaptive multi-robot patrolling through local formation transformation," in IEEE/RSJ Int Conf Intelligent Robots and Systems, pp. 1721-1728, 2016.

[28] R. Gockley, J. Forlizzi, and R. Simmons, "Natural person-following behavior for social robots," in 2nd ACM/IEEE Int Conf on HumanRobot Interaction, pp. 17-24, 2007. 